\title{
Dynamic Reliability of Buried Pressure Pipelines Subjected to Random Space-Time Earthquake Load
}

\author{
Peng Zhang ${ }^{\mathrm{a}}$, Yihuan Wang ${ }^{\mathrm{b}, *}$, and Guodong Xian ${ }^{\mathrm{c}}$ \\ ${ }^{a}$ School of Civil Engineering and Architecture, Southwest Petroleum University, Chengdu, 610500, China \\ ${ }^{b}$ School of Mechanical and Electrical Engineering, Southwest Petroleum University, Chengdu, 610500, China \\ ${ }^{c}$ Petro-China Southwest Pipeline Company, Chengdu, 610500, China
}

\begin{abstract}
Earthquake ground motion is a random process, and each response of a structure can be implemented as a random process. A large scale structure of the long buried pressure pipelines have the random variation of the space-time seismic load. A random space-time seismic load model is established by studying the time-varying and spatial characteristics of seismic load. Combined with the theory of fluctuation, the dynamic response of the buried pipeline is analyzed, and the dynamic response of the buried pressure pipeline is established by using the Von-Mises strength theory under the internal pressure. Based on the simplified formula of theoretical analysis, the first order second moment reliability method and the first transcendental failure theory are used to analyze the dynamic reliability of buried pipeline under random space-time seismic load, and the application of the method is analyzed. Dynamic reliability analysis methods for real application are validated. The feasibility of the proposed method is verified by combining the Wenchuan Earthquake. The study shows that it is necessary to consider the characteristics of random space-time seismic load when seismic damage analysis of long buried pressure pipeline, which lays the foundation for the theory of random space-time vibration.
\end{abstract}

Keywords: buried pipeline; seismic load; random space-time; dynamic reliability; first order second moment method

(Submitted on October 6, 2018; Revised on November 16, 2018; Accepted on December 18, 2018)

() 2019 Totem Publisher, Inc. All rights reserved

\section{Introduction}

Random vibration is a technology subject to study the stability, response, recognition and reliability of random structural systems with probability and statistical method [1]. The classical random vibration theory is a system based on mathematical analysis. However, it is difficult to obtain analytical solutions for linear or nonlinear structures in real engineering, and the computational burden of numerical calculation is very huge [2]. Therefore, Ou proposed the fuzzy random vibration theory and applied it to the fuzzy random dynamic reliability analysis of structures suffered from seismic load [3-4]. However, the structure with large space span is not only affected by the time-varying characteristics of seismic load, but also to the spatial correlation of seismic load. So Loh et al. used the recorded date by the SMART-1 to obtain the spatial correlation of ground motion without considering time-varying characteristics of the seismic load [5].

Rice proposed the crossover problem of random processes and fixed boundaries [6]. At present, the method of dynamic reliability analysis usually adopts the Poisson process with the assumption that the crossover times are independent [7]. To solve the problem, some scholars have proposed the improvement reliability methods based on the existing Poisson-based methods [8-9]. In general, dynamic reliability analysis is a complex integral process with huge computational burden so it is not applicable for engineering applications.

In this paper, the space-time characteristics of seismic load are coupled and the space-time correlation model of seismic loads is established to analyze the dynamic response of buried pipelines. Combining the first time beyond the destruction criteria and the first order second moment method, a suitable reliability method is proposed. 


\section{The Model of Random Space-Time Earthquake Load}

Time-varying seismic load is a non-stationary random process that is reflected by multiplying a stationary random process with a zero mean and a deterministic function with a deterministic non-stationarity [3]. That is,

$$
a(t)=\psi(t) f(t)
$$

Where:

$f(t)$ is the time-varying function of non-station of the ground motion determined by the time $t ; a(t)$ is equivalent to the seismic acceleration that changes from the beginning of the earthquake to the whole process of the attenuation; $\psi(t)$ is a smooth Guess process with mean 0 and variance 1 .

When the spatial coherence coefficient model of ground motion is substituted into Equation (1), the model of random process's acceleration with the characteristic of space-time seismic load is as follows:

$$
a(t, d)=\psi(t) f(t) \rho(d)
$$

Where:

$\rho(d)$ is the coherence function caused by the spatial change, which is equivalent to the acceleration change of the seismic acceleration with the spatial change of the whole process.

Suppose that $\psi(t)$ is smooth filtered white noise, the spectral density is [10]:

$$
S(\omega)=\frac{1+4 \zeta_{g}^{2} \frac{\omega^{2}}{\omega_{g}^{2}}}{4 \zeta_{g}^{2} \frac{\omega^{2}}{\omega_{g}^{2}}+\left(1-\frac{\omega^{2}}{\omega_{g}^{2}}\right)^{2}} S_{0}
$$

Where:

$\zeta_{g}$ is damping ratio for the characteristics of site; $\omega_{g}$ is the field of soil frequency of excellence which response peak ground motion displacement; $S_{0}$ is the spectral density of Gaussian white noise with an average of 0 .

The difference of mean and square of $\psi(t)$ can be obtained by:

$$
\sigma_{\psi}^{2}=\int_{-\infty}^{\infty} S(\omega) \mathrm{d} \omega
$$

Substitute (3) into (4):

$$
\sigma_{\psi}^{2}=\frac{\left(1+4 \zeta_{g}^{2}\right) \pi \omega_{g}}{2 \zeta_{g}} S_{0}
$$

Suppose that $\sigma_{\psi}^{2}=1 ; \quad S_{0}$ is:

$$
S_{0}=\frac{2 \zeta_{g}}{\left(1+4 \zeta_{g}^{2}\right) \pi \omega_{g}}
$$


According to the theory of random process, the ground motion is considered as a random stationary process. The seismic wave propagation direction and the self-power spectrum of the two points A and B are known. The spatial correlation of these two points is expressed by the function of cross-power spectrum as:

$$
S_{\mathrm{AB}}=\sqrt{S_{\mathrm{AA}}(\omega) S_{\mathrm{BB}}(\omega)} \rho_{\mathrm{AB}}(d, \omega) e^{-i \omega \frac{d}{v(\omega)}}
$$

Where:

$S_{\mathrm{AA}}(\omega), S_{\mathrm{BB}}(\omega)$ are self-power spectrum, $\rho_{\mathrm{AB}}(d, \omega)$ is the function of ground motion coherence of A and B; $d$ is two points distances in the earthquake; $\omega$ is seismic frequency; $v(\omega)$ is speed of visual wave.

The coherence function that defines the spatial coherence of the two points of ground motion in the frequency domain, the spatial correlation of the ground motion can be introduced into the coherence function $\rho_{\mathrm{AB}}(d, \omega)$, defined as:

$$
\rho_{\mathrm{AB}}(d, \omega)=\frac{S_{\mathrm{AB}}(\omega)}{\sqrt{S_{\mathrm{AA}}(\omega) S_{\mathrm{BB}}(\omega)}} \exp (-i \phi(d, \omega))=\left|\rho_{\mathrm{AB}}(d, \omega) \exp (-i \phi(d, \omega))\right|
$$

Where:

$S_{\mathrm{AB}}(\omega)$ is two points of A and B's power spectrum; $\left|\rho_{\mathrm{AB}}(d, \omega)\right|$ is a hysteresis-related function that measures the degree of correlation between the A and $\mathrm{B} ; i \phi(d, \omega)$ is the coherent angle that expresses effect of traveling wave.

Coherence functions can be divided into three categories: pure empirical models, semi-empirical semi-theoretical models and pure theoretical models. Hence, this paper obtained the semi-theoretical semi-empirical coherence function model, which based on the theory of spatial correlation of ground motion for seismic wave propagation in soil media of different types of sites [11]:

$$
\rho(d, \omega)=\exp \left[-\left(\beta_{1}+\beta_{2} \omega^{\beta_{3}}\right) d\right]
$$

Where:

$\beta_{1}, \quad \beta_{2}$ and $\beta_{3}$ are the three parameters of the model, respectively.

The deterministic function is proposed as the following expression [12]:

$$
f(t)=f_{m}\left[\frac{t}{c} \exp \left(1-\frac{t}{c}\right)\right]^{b}
$$

Where:

$f_{m}$ is the maximum value of $f(t) ; c$ is the corresponding time to $f_{m} ; b$ is the index of the control shape of $f(t)$.

In summary, the spectral density model of a random process of acceleration with the space-time characteristics can be expressed as:

$$
S(t, d, \omega)=S(\omega) f(t) \rho(d)
$$

The acceleration of the mean and variance values at any point is: 


$$
a_{\sigma}(z)=\left(\int_{-\infty}^{\infty} S(t, d, \omega) \mathrm{d} \omega\right)^{\frac{1}{2}}=\left(\rho(d) f(t) \int_{-\infty}^{\infty} S(\omega) \mathrm{d} \omega\right)^{\frac{1}{2}}
$$

The average value of the acceleration response maximum for any control section of a long-span structure is [13]:

$$
D_{\max }(z)=\eta a_{\sigma}(z)
$$

Where:

$\eta$ is the peak factor,$\eta=\sqrt{2 \operatorname{In} \gamma_{0} T}+\frac{0.5772}{\sqrt{2 \operatorname{In} \gamma_{0} T}} ; T$ is the duration of the earthquake; $\gamma_{0}$ is the zero-crossing rate, which is taken 3 in engineering applications.

\section{Dynamic Responses of Buried Pressure Pipelines under Random Space-Time Seismic Load}

\subsection{Dynamic Response of Pipeline under Random Space-Time Earthquake Load}

Existing studies have shown that the axial deformation of buried pipelines under seismic loading is much greater than the bending deformation [14-15]; only the axially dynamic response of pipelines is discussed in this paper. According to the related theory of seismic wave dynamics, the motion of the soil around the pipeline under the action of the seismic wave can be reduced to a harmonic plane wave [16]. The shape of corresponding wave displacement function is:

$$
Y(x, t)=A \sin 2 \pi\left(\frac{t}{T}-\frac{x}{\lambda}\right)=A \sin \left(\omega t-\frac{2 \pi x}{\lambda}\right)
$$

Where:

$\lambda$ is the wavelength, $\lambda=V_{s} T_{m}, v_{s}$ is the shear wave speed; $T_{m}$ is the characteristic period of the ground motion response spectrum; $x$ is waveform distances for seismic waves on the $\mathrm{x}$-axis; $A$ is the amplitude of the ground soil.

Assume that the angle between the propagation direction of the shear wave and the axial direction of the pipeline is $\theta$. Figure 1 shows the influence of the shear wave on the axial direction of the pipeline. The axial displacement generated by the shear wave to the ground soil is:

$$
u\left(x^{\prime}, t\right)=A \cos \theta \sin \left(\omega t-\frac{2 \pi x^{\prime}}{\lambda^{\prime}}\right)
$$

Where:

$x^{\prime}$ is the coordinates of the axis of pipeline, $x^{\prime}=\frac{x}{\cos \theta} ; \theta$ is the angle between the incident direction of the seismic wave and the axis of pipeline; $\lambda^{\prime}$ is the apparent wavelength of the shear wave in the axis direction of pipeline, $\lambda^{\prime}=\frac{\lambda}{\cos \theta} ; \zeta$ is the transfer coefficient [17], which has following expression:

$$
\zeta=\frac{1}{1+\left(\frac{2 \pi}{L}\right)^{2} \frac{E S}{u_{p} k_{1}}}
$$

Where:

$E$ is the elastic modulus of the pipeline; $S$ is the cross-sectional area of the pipeline; $\lambda$ is the wavelength of shear 
wave. $u_{p}$ is the outer surface area per unit length of the pipeline, $k_{1}$ is the elastic resistance per unit area of soil along the pipeline direction, which is $0.06 \mathrm{~N} / \mathrm{mm}^{3}$ when no test data; $L$ is the length of pipeline.

Derivation of Equation (15) on $x^{\prime}$ yields axial strain $\varepsilon_{a}$ :

$$
\varepsilon_{a}\left(x^{\prime}, t\right)=\frac{\mathrm{d} u\left(x^{\prime}, t\right)}{\mathrm{d} x^{\prime}}=-\frac{\pi A}{\lambda} \zeta \sin 2 \theta \cos \left(\omega t-\frac{2 \pi x^{\prime}}{\lambda^{\prime}}\right)
$$

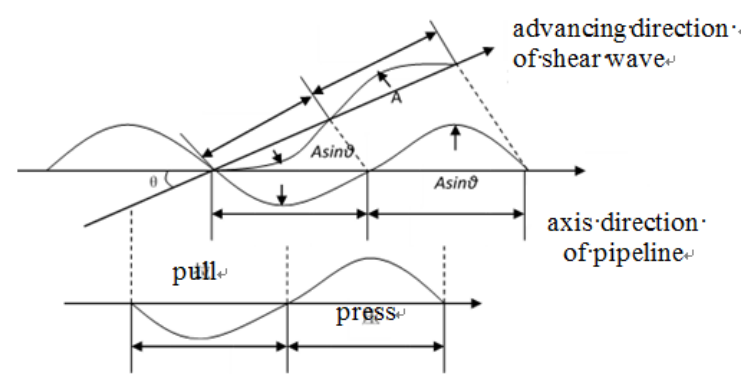

Figure 1. The effect of shear wave on the axial direction of the pipeline

When $\theta=45^{\circ}$, the axial strain reaches the maximum value. Incline angle will be more closely related to the actual effect of the earthquake on the pipeline, that is, the maximum absolute value of the axial strain:

$$
\left|\varepsilon_{a, \max }\right|=\frac{\pi A}{\lambda} \zeta \cos \left(\omega t-\frac{2 \pi x^{\prime}}{\lambda^{\prime}}\right)
$$

Using the transfer coefficient given by the specification, and Equation (18), the corresponding axial stress can be obtained as:

$$
\sigma_{a, \max }=\frac{\pi A E}{\lambda}\left[\frac{1}{1+\left(\frac{2 \pi}{\lambda}\right)^{2} \frac{E S}{K_{1}}}\right] \cos \left(\omega t-\frac{2 \pi x}{\lambda}\right)
$$

\subsection{Analysis of Dynamic Response of Buried Pressure Pipeline}

When the buried pipeline is only subjected to internal pressure, as shown as Figure 2, the stress state of the pipeline is solved by the cylindrical shell elasticity theory:

$$
\frac{\mathrm{d}^{4} w}{\mathrm{~d} x^{4}}+\frac{48}{D^{2} s^{2}} w=\frac{12\left(1-v^{2}\right)}{E s^{3}} \cdot p
$$

Where:

$s$ is current wall thickness at any point in the pipeline; $D$ is diameter; $E$ is elastic model; $v$ is Poisson's ratio; $P$ is internal pressure; $w$ is the radial displacement of any point on the pipeline wall as a function of the axial coordinate $x$.

At $x=0$, the circumferential stress is maximum and can be obtained from the above equation:

$$
\sigma_{\Phi}=\frac{P D}{2 s}
$$

Axial stress can be described as: 


$$
\sigma_{\mathrm{x}}=\frac{v P D}{2 s}
$$

Where:

$v$ is Poisson's ratio with value of 0.3 .

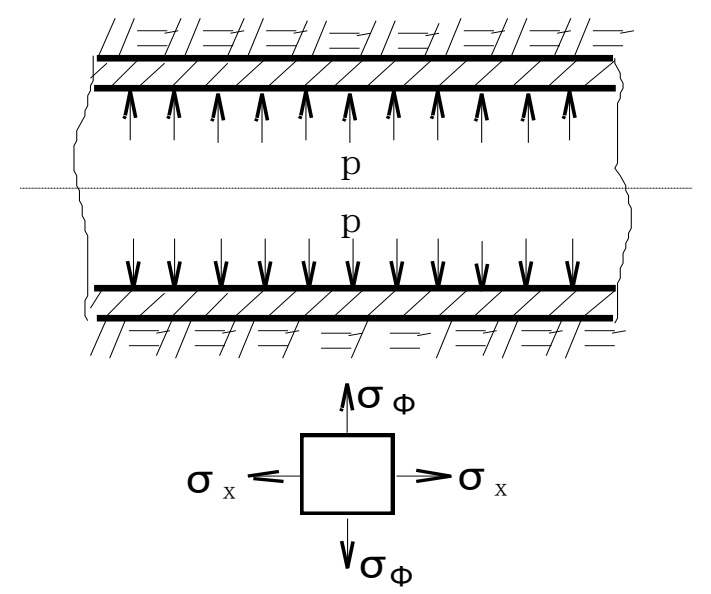

Figure 2. The stress diagram of pressure pipeline

\subsection{Dynamic Response of Buried Pressure Pipeline with Random Space-Time Earthquake Load}

When the axial stress $\sigma_{X}$ of the pipeline in internal pressure and the axial stress $\sigma_{\varphi}$ of the pipeline under seismic load act together, the stress $\sigma_{1}$ of pipeline is:

$$
\sigma_{1}=\sigma_{x}+\sigma_{e}=\frac{v P D}{2 t}+\frac{A \pi E}{\lambda}\left[\frac{1}{1+\left(\frac{2 \pi}{\lambda}\right)^{2} \frac{E S}{K_{1}}}\right] \cos \left(\omega t-\frac{2 \pi x}{\lambda}\right)
$$

Under the Von-Mises yield condition, the stress $\sigma_{s}$ of pipeline can be expressed as:

$$
2 \sigma_{s}^{2}=\left(\sigma_{1}-\sigma_{\Phi}\right)^{2}+\left(\sigma_{\Phi}-\sigma_{r}\right)^{2}+\left(\sigma_{1}-\sigma_{r}\right)^{2}
$$

Owing to the thin-walled pipeline, the radial stress is negligible, that is:

$$
\sigma_{r}=0
$$

Since there are no shear forces in the three sections above, $\sigma_{\Phi}, \sigma_{x}$ and $\sigma_{r}$ are the three principal stresses. That is:

$$
\sigma_{s}=\left(\left(\frac{P D}{2 s}\right)^{2}-\frac{P D}{2 s} \times \sigma_{1}+\sigma_{1}^{2}\right)^{\frac{1}{2}}
$$

\section{Analysis of Dynamic Reliability of Buried Pipelines}

\subsection{Basic Method}

Assuming that all the influencing parameters follow normal distributions, the limit state function of pipeline is: 


$$
Z=g(R, S)=R-S
$$

Where:

$R$ is the resistance of the pipeline; $S$ is effect of seismic load.

When $Z=0$, the structure is in the limit state; when $Z>0$, the structure is in a reliable state; when $Z<0$, the structure is in a failure state. If the mean and standard deviation of $S$ and $R$ are $\mu_{S}, \sigma_{S}$ and $\mu_{R}, \sigma_{R}$, then the reliable indicators as follows:

$$
\beta=\frac{\mu_{Z}}{\sigma_{Z}} \approx \frac{g_{X}\left(u_{X}\right)}{\left[\sum_{i=1}^{2}\left(\frac{\partial g_{X}\left(u_{X}\right)}{\partial X_{i}} \sigma_{X_{I}}\right)^{2}\right]^{\frac{1}{2}}}=\frac{u_{R}-u_{S}}{\sqrt{\sigma_{R}^{2}+\sigma_{S}^{2}}}
$$

Therefore, the reliability probability of pipeline is by the first order second moment method:

$$
P_{S}=1-P_{f}=1-\varphi(\beta)=\varphi(\beta)
$$

Where:

$P_{f}$ is the probability of failure for pipeline; $\varphi(\cdot)$ is the standard normal distribution.

\subsection{The Dynamic Reliability of Pipeline under Random Space-Time Earthquake Load}

According to the first failure criterion beyond the failure and damage criterion of the structure, the pipeline is safe within a specified time within the specified value of response; if the stress of the control point of the pipeline exceeds the safety limit, the pipeline is fail. Using the discretization of the random input process, the first order second moment method of static reliability is used to solve the problem. The steps are as follows:

- According to the fitted random space-time dynamic response curve, a series of discrete points in the random process are analyzed through software [18];

- $\mu_{S}$ and $\sigma_{S}$ are obtained after repeated calculations of the discrete points of the solved statistics.

- The limit state Equation is formed by the statistics $\mu_{R}$ and $\sigma_{R}$ for the design of the pipeline, $\mu_{S}$ and $\sigma_{S}$ are solved in step 2.

- The reliability $P_{S}$ can be obtained according to the first order second moment method in Section 4.1.

\section{The Dynamic Reliability Analysis of Lanchengyu Typical Product Segments in Wenchuan Earthquake}

\subsection{Lanchengyu Pipeline of Wenchuan Earthquake Area}

Based on the Wenchuan Earthquake in Sichuan, the dynamic reliability of the Jiangyou-Guangyuan section of the Lanchengyu pipeline in the earthquake area was analyzed. Moreover, the pipeline is X60 and the data is obtained as Table 1

\begin{tabular}{|c|c|c|c|c|}
\hline Diameter/mm & Elastic modulus/(GPa) & Wall thickness/mm & Poisson's ratio & Yield stress//(MPa) \\
\hline 457 & 207 & 8.7 & 0.3 & 0.4 \\
\hline \multicolumn{2}{|c|}{ Average shock amplitude $/\left(\mathrm{cm} / \mathrm{s}^{2}\right)$} & Shear wave/(m/s) & Frequency/(rad/s) & Damping ratio \\
\hline \multicolumn{2}{|c|}{389.43} & 283 & 20.94 & 0.72 \\
\hline
\end{tabular}
and Table 2.

Table 1. Material of pipeline 


\subsection{Analysis of Dynamic Response of Pipeline under Random Space-Time Earthquake Load}

According to the statistical time-history point distribution of the difference of mean and square $f(t)$ of the ground acceleration of the magnitude 8 [12], the curve model has following expression:

$$
f(t)=0.0544 g t e^{-0.28 t}
$$

According to the literature [20], the remarkable period of the general site is approximately $0.5 \mathrm{~s}$. The damping ratio is approximately 0.1 by the experimental data of the soil dynamic parameters in the Sichuan Basin [21]. Therefore, we have established:

$$
\sigma_{a, \max }=\left[117676-32981 \sqrt{t e^{-\left(0.28 t+5.02 \times 10^{-4} x\right)}} \cos (12.57 t-0.016 x)+45014 t e^{-\left(0.28 t+5.02 \times 10^{-4} x\right)} \cos ^{2}(12.57 t-0.016 x)\right]^{\frac{1}{2}}
$$

When studying the earthquake time is $0-20 \mathrm{~s}$ and the relative distance is $0-10 \mathrm{~km}$. The dynamic response of the buried pipeline is shown in Figure 3.

The result shows that the mean value of stress $u_{S}$ is $364.14 \mathrm{MPa}$ and the standard deviation $\sigma_{S}$ is $36.88 \mathrm{MPa}$. According to the method introduced in Section 4.2, $\beta$ is 1.84 . With the assumption of normal distribution, the reliability probability $P_{S}$ is 0.9671 .

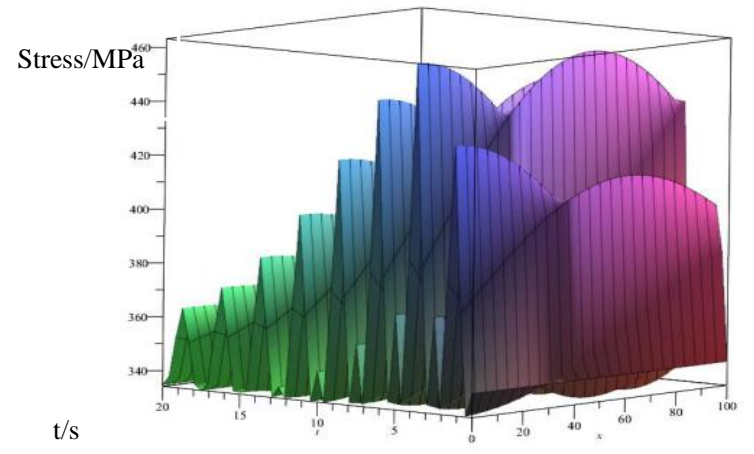

Figure 3. Dynamic response map of random space-time buried pipeline during strong earthquake

\subsection{Comparison of Dynamic Reliability of Buried Pipeline based on Different Calculation Theory}

Time-varying seismic load response of the buried pipeline were calculated using a separate random spatial load model. The reliability was compared with the dynamic reliability of the random space-time model.

\subsubsection{Dynamic Reliability of Buried Pipeline based on Time-Varying Earthquake Load}

The time-varying model of seismic load can be obtained from Equation (2), Equation (3) and Equation (11) as:

$$
S(t, \omega)=\frac{1+4 \zeta_{g}^{2} \frac{\omega^{2}}{\omega_{g}^{2}}}{4 \zeta_{g}^{2} \frac{\omega^{2}}{\omega_{g}^{2}}+\left(1-\frac{\omega^{2}}{\omega_{g}^{2}}\right)^{2}} \cdot \frac{2 \zeta_{g}}{\left(1+4 \zeta_{g}^{2}\right) \pi \omega_{g}} \cdot f_{m}\left[\frac{t}{c} \exp \left(1-\frac{t}{c}\right)\right]^{b}
$$

Combining with Equation (12), the mean value of the maximum acceleration response for any control section of a long-span structure is: 


$$
D_{\max }(z)=\eta a_{\sigma}(z)=\left(\sqrt{2 \operatorname{In} \gamma_{0} T}+\frac{0.5772}{\sqrt{2 \operatorname{In} \gamma_{0} T}}\right)\left(f(t) \int_{-\infty}^{\infty} S(\omega) \mathrm{d} \omega\right)^{\frac{1}{2}}
$$

Combined with Section 3.1, it can be derived that the dynamic response of the pipeline under time-varying seismic load is:

$$
\sigma_{a, \max }=\frac{a(z) T_{m}^{2} \pi E \xi}{4 \pi^{2} \lambda} \cos \left(\frac{2 \pi x}{\lambda}\right)
$$

Combined with Sections 3.2 and 3.3, the dynamic response of a pressure pipeline under time-varying seismic load can be derived as:

$$
\sigma_{s}=\left(\left(\frac{P D}{2 t}\right)^{2}-\frac{P D}{2 t}\left(\frac{a(z) T_{m}^{2} \pi E \xi}{4 \pi^{2} \lambda} \cos (\omega t)+\frac{v P D}{2 t}\right)+\left(\frac{a(z) T_{m}^{2} \pi E \xi}{4 \pi^{2} \lambda} \cos (\omega t)+\frac{v P D}{2 t}\right)^{2}\right)^{\frac{1}{2}}
$$

Combined with the relevant data in Section 4 and Equation (31), the dynamic response of the pressure pipeline under time-varying seismic load can be obtained as:

$$
\sigma_{s}=\left[117676-32981 \sqrt{t e^{-0.28 t}} \cos (12.57 t)+45014 t e^{-0.28 t} \cos ^{2}(12.57 t)\right]^{\frac{1}{2}}
$$

The stress curve is shown in Figure 4. The curve was processed in software and 0-20s was taken as the calculated value. The mean value of point stress $u_{s}$ is $382.77 \mathrm{MPa}$, and the standard deviation $\sigma_{s}$ is $23.44 \mathrm{MPa}$. According to the method introduced in section 4.2, $\beta$ is 2.10 . With the assumption of normal distribution, the reliability $P_{S}$ is 0.9821 .

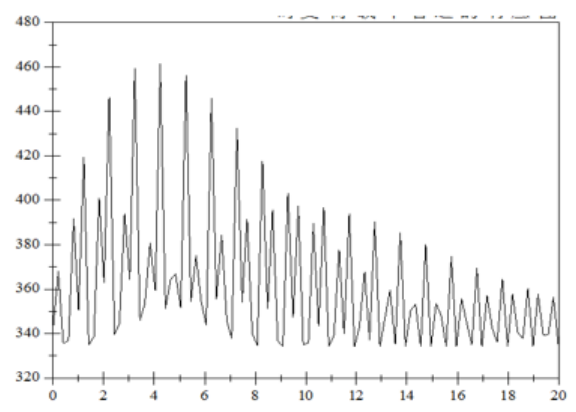

Figure 4. Stress change diagram of pressure pipeline under the action of time-varying load

\subsubsection{Dynamic Reliability of Buried Pressure Pipeline based on Space Earthquake Load}

Combined with Section 3.1, the dynamic response of the pipeline under random space earthquake load can be derived as:

$$
\left|\sigma_{a, \max }\right|=\frac{a \rho(d) T_{m}^{2} \pi E \xi}{4 \pi^{2} \lambda} \cos \left(\chi-\frac{2 \pi x}{\lambda}\right)
$$

Combined with Sections 3.2 and 3.3, the dynamic response of a pressure pipeline under random space earthquake load can be derived as:

$$
\sigma_{s}=\left(\left(\frac{P D}{2 t}\right)^{2}-\frac{P D}{2 t}\left(\frac{a \rho(d) g T_{m}^{2} \pi E \xi}{4 \pi^{2} \lambda} \cos \left(\chi-\frac{2 \pi x}{\lambda}\right)+\frac{\nu P D}{2 t}\right)+\left(\frac{a \rho(d) g T_{m}^{2} \pi E \xi}{4 \pi^{2} \lambda} \cos \left(\chi-\frac{2 \pi x}{\lambda}\right)+\frac{v P D}{2 t}\right)^{2}\right)^{\frac{1}{2}}
$$


Where:

$\chi$ is $\chi=\omega t, t$ for the time point corresponding to the peak value of the entire earthquake acceleration.

Combined with the relevant data in Section 4 and Equation (31), the dynamic response of the pressure pipeline under random space earthquake load can be obtained as:

$$
\sigma_{s}=\left[117676-17860 e^{-5.02 \times 10^{-4} x} \cos (223-0.016 x)+13456 e^{-10.04 \times 10^{-4} x} \cos ^{2}(223-0.016 x)\right]^{\frac{1}{2}}
$$

The stress curve is shown in Figure 5. The curve is processed in software and takes $0 \sim 100 \mathrm{~km}$ as the calculated value. The mean value of point stress $u_{S}$ is $365.78 \mathrm{MPa}$ and the standard deviation $\sigma_{S}$ is $14.61 \mathrm{MPa}$. According to the method introduced in Section 4.2, $\beta$ is 4.52. With the assumption of normal distribution, the reliability $P_{S}$ is 1 .

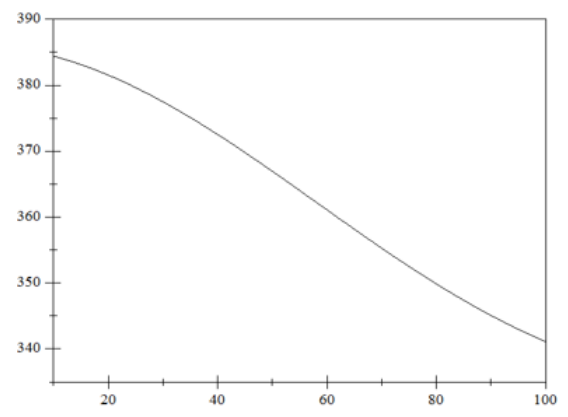

Figure 5. Stress variation diagram of pressure pipeline under random space seismic load

\subsubsection{Comparison and Analysis of Dynamic Reliability of Three Load Models}

It can be seen from Figure 6 that the same study of the pipeline, which the reliability under the random space alone is higher than that reliability under the random space-time effect, only considers the attenuation of the load with position change of the pipeline. While the reliability of time varying is higher than that under random space-time effect, the method only uses the quasi-static calculation of the wave theory, which does not consider that the buried pipeline is different from the "point" structure. Moreover, the spatial correlation of ground motions is also very sensitive. In summary, combining load analysis with time and space is the most reasonable to analyze the reliability of buried pipeline under the action of seismic load.

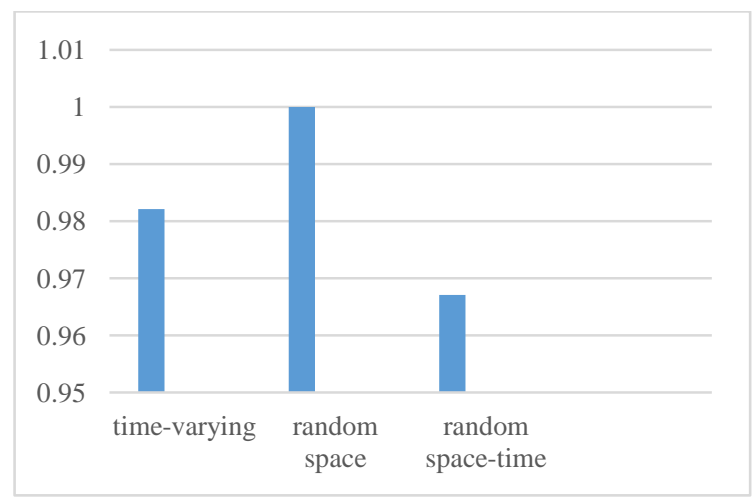

Figure 6. Comparison of the reliability the three methods

\section{Conclusions}

This paper analyzes the dynamic reliability of buried pressure pipeline under random space-time earthquake load. The main contents include the combination of seismic load as a random time and random space to establish a random space-time vibration model. Therefore, the specific conclusions are as follows:

1) By combining the linear distribution characteristics of buried pipeline, it found that seismic load has spatial randomness and time randomness. 
2) The proposed method considers the spatial effects of long-span structures under seismic load. This paper takes any of the segments as the research object, so the result of the example is approximate the value. Assuming that the parameters of the buried soil do not change, and the actual types of site soil change, the reliability value should also have a reduction factor based on this calculation.

3) Based on the existing research results, a mathematical model of random space-time seismic load is established. However, most of the models are based on classical semi-empirical semi-theoretical models. In the specific application, the coefficient correction must apply to local specific seismic data.

4) Using random process theory to derive the dynamic response of the pressure pipeline under the random space-time earthquake is more suitable for pipeline engineering's application. The first damage principle based on dynamic reliability simplifies the problem of the reliability of buried pipeline in four-dimension, which is more suitable for evaluating the popularization and application of the dynamic reliability of pressure pipeline engineering under earthquake load.

\section{Acknowledgements}

Authors gratefully acknowledge the financial support provided by the National Natural Science Foundation of China (Grant No. 50974105) and the Research Fund for the Doctoral Program of Higher Education of China (Grant No.20105121110003).

\section{References}

1. W. Q. Zhu, "Random Vibration," Science Press, Beijing, 1992

2. Z. J. Liu, "Research Progress of Seismic Analysis Method based on Random Vibration Theory," Earthquake Engineering and Engineering Vibration, Vol. 26, No. 4, pp. 47-51, 2006

3. J. P. Ou, "Fuzzy Random Vibration," Harbin Institute of Architecture and Engineering Doctoral Dissertation, Harbin, 1987

4. G. Y. Wang and J. P. Ou, "Theory and Application of Structural Fuzzy Random Vibration," Journal of Harbin Institute of Civil Engineering and Architecture, No. 2, pp. 28-41, 1986

5. C. H. Loh, "Analysis of the Spatial Variation of Seismic Waves and Ground Movements from Smart-1 Array Data," Earthquake Engineering \& Structural Dynamics, Vol. 13, No. 5, pp. 561-581, 2010

6. S. O. Rice, "Mathematical Analysis of Random Noise," Bell System Technical Journal, Vol. 24, pp. 52-162, 1944

7. N. Liu and G. T. Liu, "Time-Dependent Reliability Assessment for Mass Concrete Structures," Structural Safety, Vol. 21, No. 1, pp. 23-43, 1999

8. A. Naess, "Crossing Rate Statistics of Quadratic Transformations of Gauss Processes," Probabilistic Engineering Mechanics, Vol. 16, No. 3, pp. 209-217, 2001

9. Z. H. Zhang and W. J. Yang, "The Development and Research of Structural Dynamic Reliability Theory," Spatial Structure, Vol. 18, No. 4, pp. 64-75, 2012

10. R. Kraff, "Structural Dynamics," Higher Education Press, 2006

11. Q. J. Chen, Y. S. Yang, and Y. C. Wang, "A Practical Model for Spatial Coherent Function of Ground Motion based on Strong Seismic Array Records," China Civil Engineering Journal (s1), 2016

12. G. Y. Wang, "Analysis Method of Structural Response under Non-Stationary Strong Earthquake," China Civil Engineering Journal, No. 1, pp. 16-24, 1964

13. A. G. Davenport, "Note on the Distribution of the Largest Value of a Random Function with Application to Gust Loading," Proceedings of the Institute of Civil Engineers, Vol. 28, No. 2, pp. 187-196, 1964

14. A. Hindy and M. Novak, "Earthquake Response of Underground Pipelines," Earthquake Engineering \& Structural Dynamics, Vol. 7, No. 5, pp. 451-476, 2010

15. A. Zerva, H. S. Ang, and Y. K. Wen, "Lifeline Response to Spatially Variable Ground Motions," Earthquake Engineering \& Structural Dynamics, Vol. 16, No. 3, pp. 361-379, 2010

16. H. Yu, P. C. Li, and Z. M. Li, "Maximum Seismic Response of Buried Pipelines under Action of Seismic Traveling Waves," Special Structures, Vol. 24, No. 3, pp. 1-3, 2007

17. GB50032-2003, "Code for Seismic Design of Outdoor Water Supply, Drainage and Gas Heat Engineering," 2003

18. X. L. Ju, "Research on Random Wind-Induced Response and Time-Dependent Reliability of Suspension Crossing Pipes," Daqing Petroleum Institute, 2008

19. Y. C. Li and Q. J. Chen, "Analysis of Near-Field Vibration Characteristics based on Wenchuan M8.0 Strong Earthquake Record," Journal of Catastrophology, Vol. 24, No. 3, pp. 1-3, 2012

20. Z. Y. Zhang and B. T. Sun, "Study on the Model Parameters of the Seismic Power Spectrum for the New Seismic Code," World Earthquake Engineering, Vol. 16, No. 3, pp. 33-38, 2000

21. B. X. Shi and Y. J. Zhou, "Statistical Analysis of Site Dynamic Parameters in the Sichuan Basin," Earthquake in Sichuan, No. 3 , pp. 1-5, 2014 